\title{
¿CÓMO HACER UNA BASE DE DATOS OCUPANDO UNA PLANILLA DE CÁLCULO DE EXCEL?
}

\author{
HOW TO MAKE A DATABASE USING AN EXCEL SPREADSHEET?
}

\section{Dr. Gerardo Weisstaub}

Profesor asistente. Departamento de Pediatría (Campus Centro) de la Universidad de Chile. Instituto de Nutrición y Tecnología de los Alimentos (INTA).

\section{INTRODUCCIÓN}

Crear la base de datos (BD) para almacenar los datos de un estudio, es un paso fundamental antes de iniciar el análisis de los datos. Aunque existen gran cantidad de software estadísticos (ej.: Stata (1)) y sitios web (ej.: RedCap (2)) que permiten generar $\mathrm{BD}$, en este apartado nos enfocaremos en cómo realizar una BD en una hoja de cálculo de Microsoft Excel. Hemos decidido explicar la ocupación de este programa porque que es el más difundido, tiene una gran cantidad de pruebas estadísticas y los archivos creados pueden ser exportados a los otros programas de estadística. Nos parece importante resaltar que nos enfocaremos en explicar los aspectos básicos para poder ocupar una hoja de Excel como base de datos, por lo que no utilizaremos opciones de programación o interpretación de datos que tiene el software.

\section{ALGUNAS CONSIDERACIONES GENERALES}

\section{Diseño de la planilla de cálculo}

Se deben colocar en las columnas las variables y en las filas los datos de cada sujeto.

\section{Orden de las variables en la base de datos}

Es una buena práctica que en toda base de datos la primera columna sea un identificador común (ID) con el que se reconozca a un mismo sujeto en todos los documentos 0 archivos en los que se registren sus datos. El resto de las variables deberían tener un orden congruente. Por ejemplo, colocar las variables demográficas juntas, luego todas las variables clínicas y así sucesivamente.

\section{Definir las variables}

Antes de iniciar la construcción del BD es fundamental tener muy presentes los objetivos del proyecto de investigación y las variables que se desean medir para poder cumplir con ellos. Recordemos que "una variable es una característica o propiedad del sujeto (u objeto) en estudio y que puede ser medida 0 cuantificada de una o diversas formas" (3).

A veces ocurre que al momento de querer construir la base de datos nos damos cuenta que algunos de los objetivos o las variables

\section{RESUMEN}

Una de las etapas fundamentales en el desarrollo de un protocolo de investigación es la construcción de la base de datos. Su diseño detallado y el llenado cuidadoso ayudan a mejorar la confiabilidad de los resultados obtenidos a partir del análisis estadístico de los datos ingresados. Justamente, uno de los problemas que frecuentemente deben enfrentar los profesionales de la salud, es la construcción y la digitación de los datos. Procesos que, de no ser realizados adecuadamente, pueden generar diferentes tipos de errores. Aunque existen softwares específicos para el armando de las bases de datos, no es frecuente que los profesionales de la salud sepan utilizarlos. Es por lo anterior que el objetivo de este apartado es describir algunos aspectos básicos del uso de la planilla de cálculo de Excel para que pueda ser utilizada como base de datos.

Palabras claves: Base de datos, Software estadístico, Fiabilidad.

\section{ABSTRACT}

One of the fundamental stages in the development of a research protocol is the construction of the database. Its detailed design and careful filling help improve the reliability of the results obtained from the data's statistical analysis. One of the problems that health professionals must frequently face is the construction and entry of data. Processes that, if not carried out properly, can generate different types of errors. Although there is a specific software for building databases, health professionals do not know how to use them. Because of the above, the objective of this section is to describe some essential aspects of the use of the Excel spreadsheet so that it can be used as a database.

Keywords: Database; Software Tools; Reliability.

que nos hemos planteado requieren ser modificados, por lo que puede ser útil construir la base de datos junto al proyecto de investigación. Por ejemplo, es un estudio en el que se intentó conocer la frecuencia de fobia a la fiebre por parte de los padres, al intentar incluir en la BD las variables que permitirían saber cuándo la madre de un niño tenía fobia a la fiebre (ejemplo: temperatura, uso de medicación) los autores se dieron cuenta que esta variable no estaba claramente definida.

Supongamos que el objetivo del proyecto es evaluar la asociación que existe entre la duración de la lactancia materna en menores de un año y el uso del chupete. Algunas de las variables que se derivan de este objetivo son: duración de la lactancia materna, uso del chupete, edad de inicio del uso del chupete, situaciones en que se ocupa el chupete, edad de la madre, tiempo de uso diario del chupete.

\section{Tipo de variables}

Antes de incorporar esas variables a la base, debemos recordar la clasificación de las variables. Estas pueden ser cuantitativas (ej.: edad) o cualitativas (ej.: sexo). Las primeras pueden dividirse en cuantitativas discretas, en la que no existen valores intermedios como el número de hijos (ej: 10 2) o continuas, en que si es posible definir valores intermedios como en el caso de la talla (ej.: 160 o 1610162 cm) (4).

\section{Nombre de las variables}

El nombre de cada variable debe ser claro, corto y lo más representativo posible. Se sugiere hacer, en otra hoja de la misma planilla de cálculo, una lista con los códigos de cada variable en el que se describa claramente que representa cada uno. Estos nombres pueden tener letras y/o números y/o guion bajo, pero se sugiere evitar acentos, espacios o caracteres especiales que dificulten su identificación al exportar la planilla de cálculo a otro software. Si una misma variable es ocupada más de una vez en el mismo estudio (ejemplo: peso) se sugiere colocar un numero al final (ejemplo: peso1, peso2, peso3).

\section{Autor para correspondencia:} Dr. Gerardo Weisstaub E-mail: gweiss@inta.uchile.cl 
Definir las categorías o límites de las variables

En la misma hoja en la que se listen los códigos de la variable se sugiere definir las categorías o los límites de cada una. Por ejemplo, en el caso de una variable cualitativa como "educación", esta puede ser categorizada como "primaria", "secundaria", "terciaria". Si es una variable cuantitativa, debe definirse la unidad en la que será expresada (ej: "Kg" en el caso de peso) y los limites considerados normales (ej.: 3000 a $4500 \mathrm{~g}$ en el caso del peso al nacer).

\section{Digitación cuidadosa}

Cada vez que se ingresa un dato, en papel 0 en un formato electrónico, existe riesgo de que el dato sea mal ingresado. Aunque es poco probable que logremos evitar cometer un error al momento de ingresar el dato a nuestra base, se pueden diseñar algunas estrategias para reducir la probabilidad de equivocarse. Una opción es digitar el valor de la variable dos veces. Por ejemplo, si la edad del sujeto es de 50 años y se digita 500, es menos probable que este mismo error se cometa de la misma manera la segunda vez que se digita (la diferencia entre ambos valores se comporta como un llamado de atención para verificar el valor correcto). Otra opción que existe en algunos softwares (ejemplo: Excel) es colocar límites al valor de la variable. Por ejemplo, podemos definir que el límite de talla de los sujetos de nuestro estudio varía entre 100 y $160 \mathrm{~cm}$, por lo que si intentamos ingresar el valor 170 el programa no lo permitirá.

\section{Limpieza de la base de datos}

Antes de realizar el análisis de nuestros datos es importante asegurarse de su calidad. Aunque se tomen medidas para evitar al máximo el ingreso de datos erróneos, las tasas de error en las bases de datos se encuentran generalmente entre el $5 \%$ o más. Es necesario entonces realizar un conjunto de operaciones que permitan identificar omisiones, datos incompletos, incorrectos o incoherentes para corregirlos o eliminarlos (ejemplo: una niña que mide al inicio del estudio menos de lo que mide al final). Se pueden ordenar los datos de una variable de menor a mayor y revisar si existen datos que estén muy lejos de los considerados normales u obtener promedios y desviaciones estándar y revisar los datos que estén más allá de \pm 3 desviaciones. En resumen, aunque esta revisión requiere mucho trabajo, es fundamental, ya que, si nuestra base datos tiene gran cantidad de datos "basura", solo podremos obtener basura como resultado (5).
Almacenamiento y nombre de la base de datos

Sugiero guardar siempre la base "original" y realizar la limpieza en una copia, ya que si se comete un error al limpiarla siempre tendremos la original. Es muy importante mantener el orden con el que se almacenan las bases de datos. Suele ocurrir que cuando tenemos más de un archivo estos se guardan en distintas carpetas o con nombres diferentes, especialmente si los ocupan diferentes personas. Existen diferentes opciones para mantener actualizada y ordenada la base de datos. Por ejemplo, guardarla en una carpeta compartida y que las diferentes personas solo ocupen esa base. Lo ideal es que solo una persona sea responsable de realizar los cambios en la BD.

\section{Anonimato}

Es importante considerar que cada vez que se comparte una base de datos estas deben estar anonimizadas, por lo que no pueden contener el nombre, documento de identidad ni dirección de las personas a las que pertenecen los datos. Se sugiere que solo el investigador principal del proyecto guarde la base con los datos completos de los sujetos para que se puedan contactar con ellos en caso de ser necesario.

Para aprender a diseñar esta base de datos he diseñado un ejercicio que se detalla a continuación. Se planifica un estudio cuyo objetivo es conocer el estado nutricional de un grupo de niños que asisten a las escuelas de la ciudad donde vivimos. Seguir las siguientes instrucciones paso a paso.

Lo primero que hacemos el hacer el listado de las variables que necesitamos registrar, sus códigos, clasificación y límites (con los datos registrados evaluaremos el estado nutricional en un software específico). Dependiendo de quien tenga acceso a esta base de datos se podrían agregar los datos para identificar a los niños y niñas del estudio.

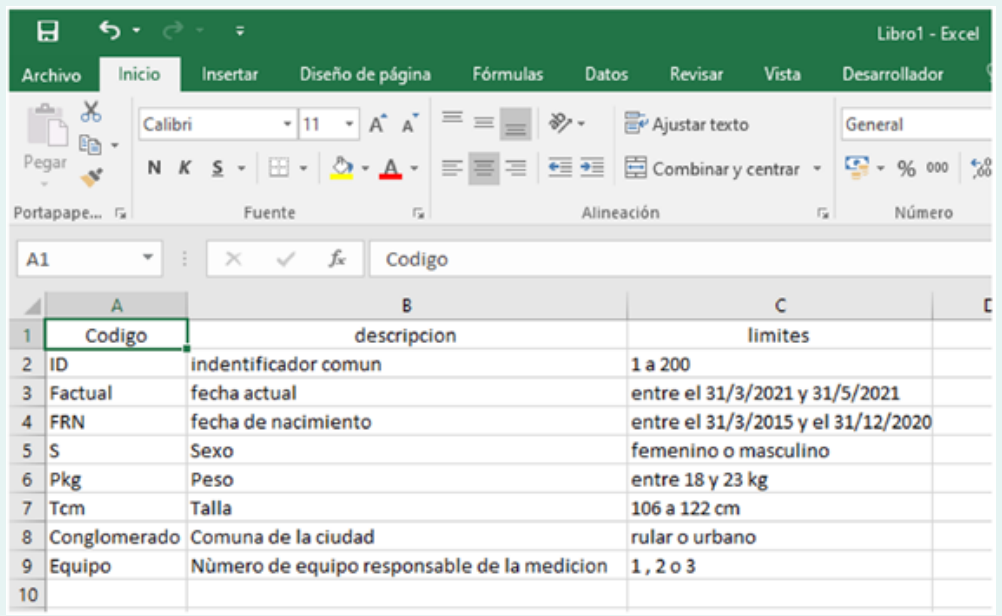

Llevamos la variable definida a la otra hoja de cálculo del mismo libro del archivo Excel. Recordar que la primera variable es "ID" (el identificador común) y luego colocamos el resto de las variables en las siguientes columnas.

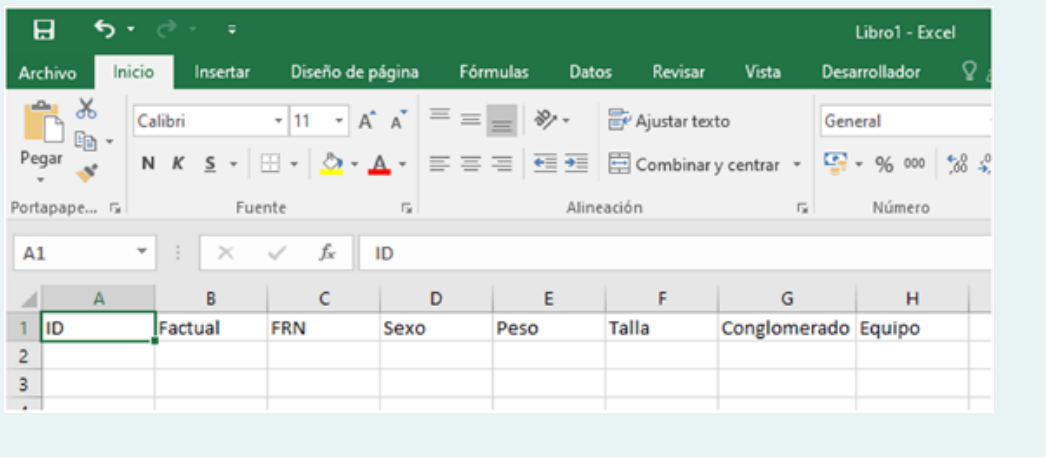


Dado que ya conocemos la clasificación o límites de las variables ahora vamos a establecer esos límites en las celdas donde ingresaremos los datos para disminuir el riesgo de equivocarnos

Empezaremos por colocar los límites del ID (entre 1a 200). Colocamos el curso en la celda que está debajo de la variable (ver rectángulo enmarcado en verde), seleccionamos la pestaña "Datos" y luego donde dice "validación de datos (como aparece en la figura).

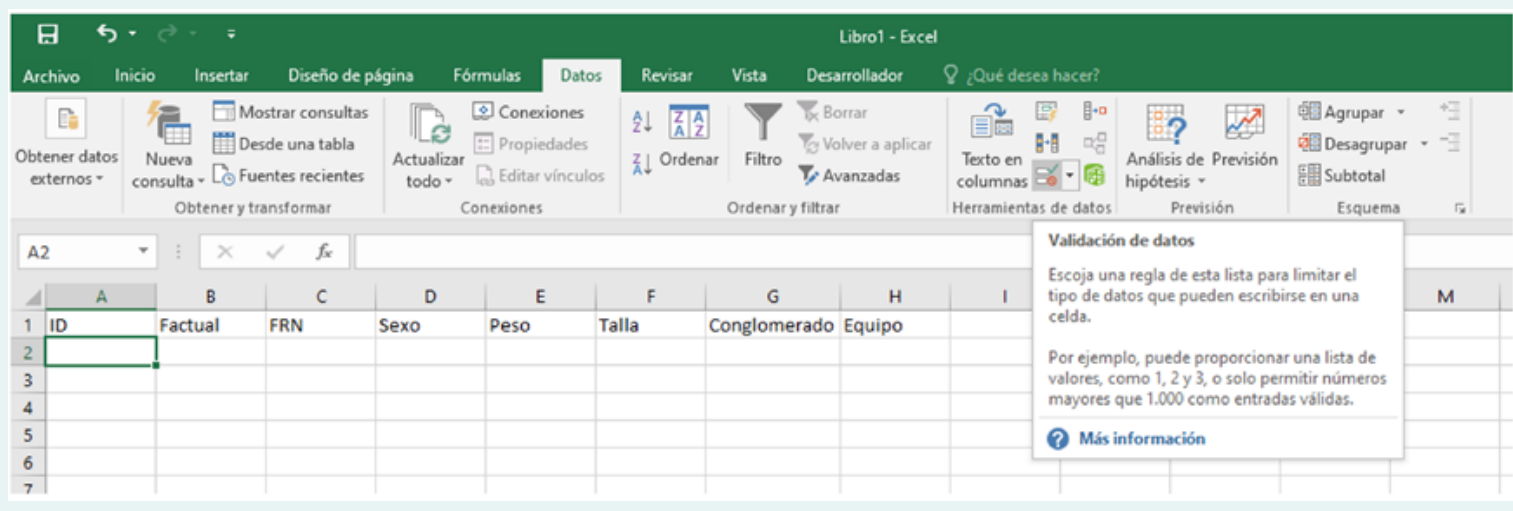

Al cliquear sobre "validación de datos "aparecerá una ventana como la siguiente.

\begin{tabular}{|c|c|c|c|}
\hline \multicolumn{4}{|l|}{ Validación de datos } \\
\hline Configuración & Mensaje & de entrada & Mens: \\
\hline \multicolumn{2}{|c|}{ Permitir: } & & \\
\hline \multicolumn{2}{|l|}{ Cualquier valor } & \multicolumn{2}{|c|}{$\nabla$ Omitir blancos } \\
\hline \multicolumn{4}{|l|}{ Datos: } \\
\hline entre & $\checkmark$ & & \\
\hline
\end{tabular}

Aplicar estos cambios a otras celdas con la misma configuración

Dado que el valor en esta celda oscilara entre 1 a 200 (numero entero) elegimos esa opción, completamos el valor mínimo y máximo.

\begin{tabular}{|c|c|c|c|c|}
\hline \multicolumn{4}{|c|}{ Validación de datos } & \multirow[t]{2}{*}{$?$} \\
\hline Configuración & Mer & saje de entrada & Mensaje de error & \\
\hline \multicolumn{5}{|c|}{ Criterio de validación } \\
\hline \multicolumn{5}{|c|}{ Permitir: } \\
\hline \multicolumn{2}{|c|}{ Número entero } & \multicolumn{2}{|c|}{$\nabla$ Omitir blancos } & \\
\hline \multicolumn{5}{|l|}{ Datos: } \\
\hline \multicolumn{5}{|l|}{ entre } \\
\hline \multicolumn{5}{|l|}{ Minimo: } \\
\hline \multicolumn{3}{|l|}{$\overline{1}$} & है: & \\
\hline \multicolumn{5}{|l|}{ Máximo: } \\
\hline \multicolumn{3}{|l|}{200} & हक: & \\
\hline \multicolumn{5}{|c|}{$\square$ Aplicar estos cambios a otras celdas con la misma configuración } \\
\hline \multicolumn{3}{|l|}{ Borrar todos } & Aceptar & Cancelar \\
\hline
\end{tabular}

Cliqueamos al lado de donde dice "Cualquier valor" y aparecerán las siguientes opciones.

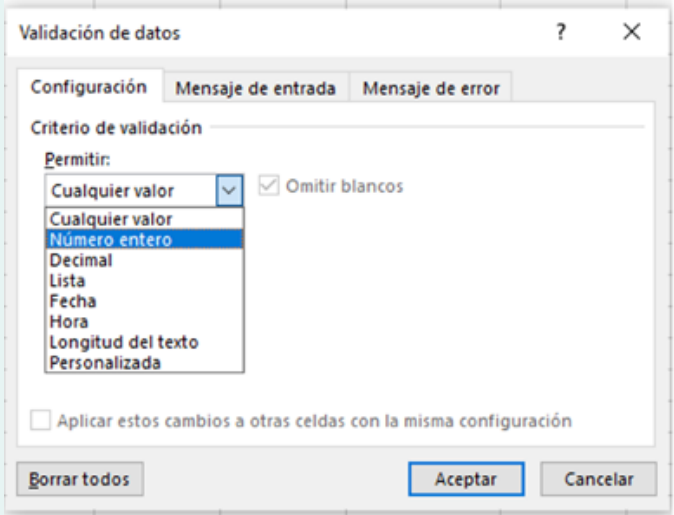

7

Luego, sin cliquear aun en la opción aceptar, cliqueamos en la ventana que de "Mensaje de error" y completamos la opción de "Titulo" y el "Mensaje de error" (de modo de ayudar a la persona que digita el dato) y posteriormente cliqueamos en "Aceptar".

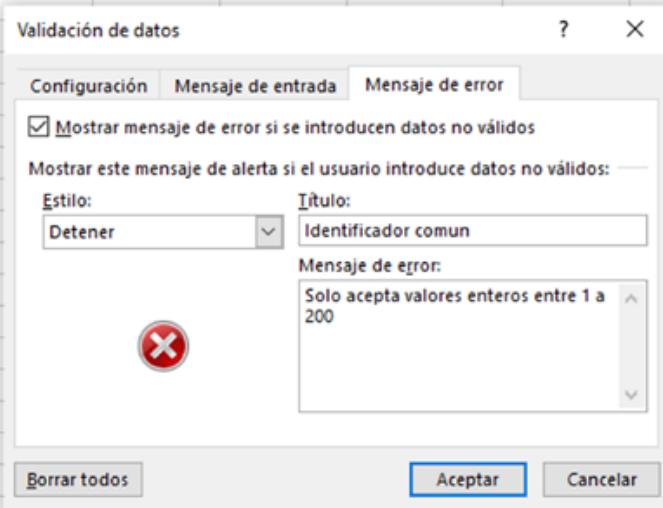


8

Si ahora intenta ingresar un valor menor a 10 superior a 200 aparecerá un mensaje de error.

\begin{tabular}{l} 
Identificador comun \\
Solo acepta valores enteros entre 1 a 200 \\
Reintentar \\
Cancelar \\
\hline Ayugda \\
\hline
\end{tabular}

Ahora veamos un ejemplo con una variable cualitativa como "sexo

1

Primero debemos escribir las opciones que ocuparemos para clasificar la variable en otro

lugar de la base (ver celda "J1" y "J2) y luego da en verde) debajo de la variable. colocamos nuestro cursor (recuadro de la cel-

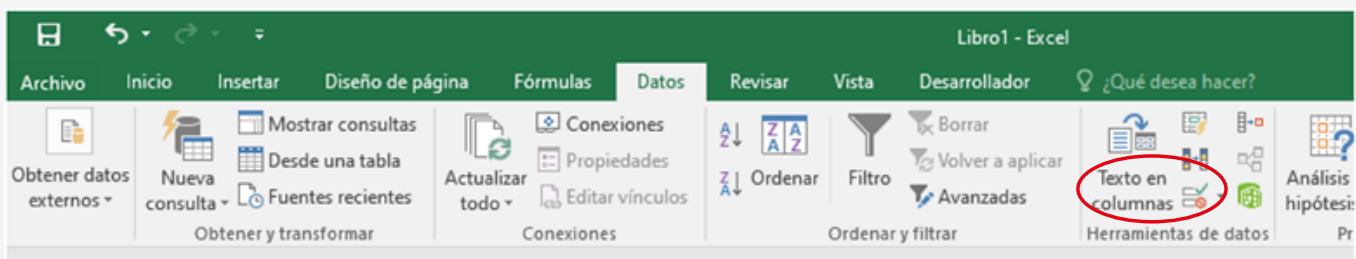

\begin{tabular}{|c|c|c|c|c|c|c|c|c|c|c|c|c|c|}
\hline D2 & & $v$ & $\vdots$ & $x$ & $\checkmark$ & $f_{x}$ & & & & & & & \\
\hline 4 & A & & & B & & C & D & $E$ & $\mathrm{~F}$ & G & H & 1 & $J$ \\
\hline 1 & ID & & Factua & & FRN & & Sexo & Peso & Talla & Conglomerado & Equipo & & femenino \\
\hline 2 & & & & & & & & & & & & & masculino \\
\hline
\end{tabular}

Ahora volvemos a cliquear en la ventana de "Datos" y luego en "Validación de datos" (donde indica la flecha roja) y en esta oportunidad seleccionamos la opción "Lista".

\begin{tabular}{l} 
Validación de datos \\
Configuración Mensaje de entrada Mensaje de error \\
Criterio de validación \\
Permitir: \\
\hline Dasta \\
\hline entos: \\
\hline Origen: \\
\hline Aplicar estos cambios a otras celdas con la misma configuración desplegable \\
\hline Borrar todos \\
\hline
\end{tabular}

Luego de cliquear en la opción "Origen" (donde indica la flecha roja) seleccionamos con nuestro cursor las celdas donde se encuentra las opciones de la variable (líneas punteadas en verdes). Automáticamente se llenarán los valores de las celdas para validar la variable en el cartel "Validación de datos".

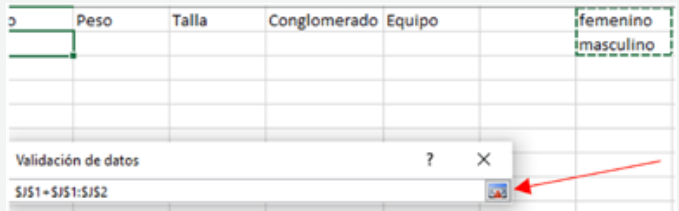

Volvemos a cliquear en el lugar que indica la flecha roja y por último cliqueamos en la opciòn "Aceptar". 
4

Ahora cuando querramos ingresar el "sexo" en esa columna solo apareceran las opciones que hemos seleccionado.

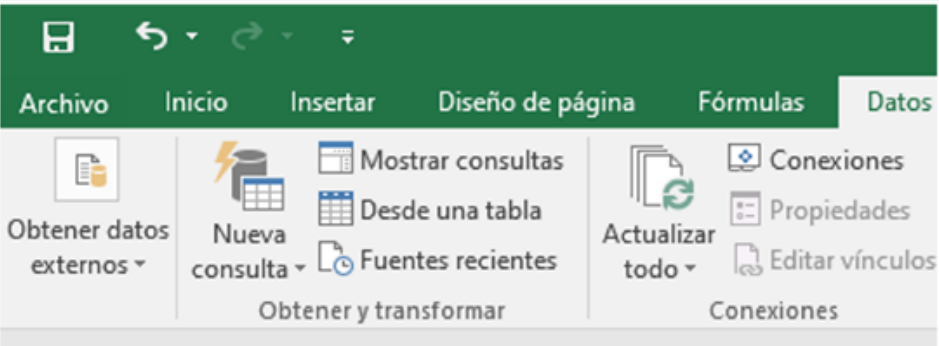

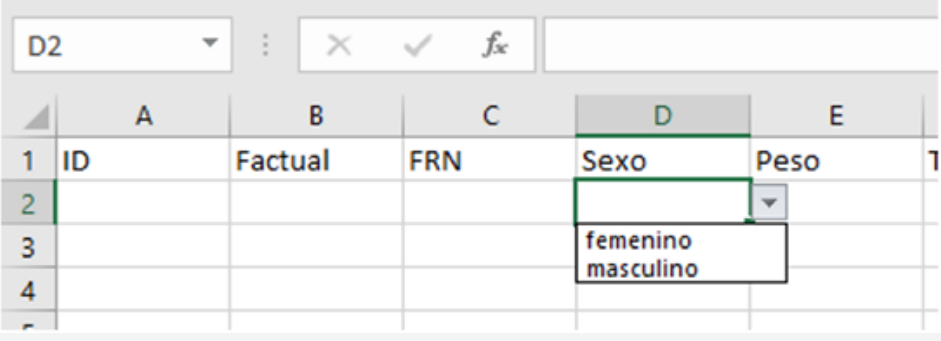

Luego de completar la validación de todas las variables en la primera fila, copiamos esas celdas y las pegamos hasta a la fila que seleccionemos (en este caso hasta la fila 201)

Finalmente guardamos nuestra planilla de cálculo trasformada en base de datos.

\section{CONCLUSIONES}

El diseño y el llenado cuidadoso de una base de datos es una etapa indispensable de cualquier protocolo de investigación. Su construcción es una oportunidad para revisar la definición, el tipo de variables y su clasificación, aspectos que deben ser descritos con detalle en la metodología de un protocolo. Utilizar filtros adecuados durante el llenado de la base puede ser importante para mejorar la confiabilidad de los datos y los resultados obtenidos a partir de ellos. Es importante considerar que la digitación, almacenamiento y limpieza previa al análisis son etapas que no pueden ser omitidas. Por último, se debe recordar que toda base datos que se comparte debe estar anonimizada de modo de resguardar adecuadamente la privacidad de los datos.

Conflicto de interés: El autor declara no presentar conflicto de interés.
REFERÊNCIAS

1. Página web https://www.stata.com/ (acceso febrero 2021)

2. Página web https://www.project-redcap.org/ (acceso febrero 2021)

3. Fuentes A. M, Yohannessen V K. Como leer y generar publicaciones científicas. Rol y definición de las variables en una investigación: el protagonismo que se merecen. Neumol Pediatr 2019;14(3):122-5. DOI https://doi. org/10.51451/np.v14i3.97

4. Villasís-Keever MA, Miranda-Novales MG. El protocolo de investigación IV: las variables de estudio. Rev Alerg Mex. 2016:63(3):303-310

5. Serie de cuadernillo técnico. Limpieza de base de datos (Internet). Dirección General de Evaluación e Investigación Educativa- 2015. Ministerio de Educación de Guatemala. Disponible en https://www.mineduc.gob.gt/ digeduca/documents/cuadernillosTecnicos/Limpieza_Bases_de_Datos.pdf 